\title{
Novel enzymatically cross-linked hyaluronan hydrogels support the formation of 3D neuronal networks
}

\section{Journal Article}

Author(s):

Broguiere, Nicolas; Isenmann, Luca; Zenobi-Wong, Marcy (iD

Publication date:

2016-08

Permanent link:

https://doi.org/10.3929/ethz-b-000117146

Rights / license:

Creative Commons Attribution-NonCommercial-NoDerivatives 4.0 International

Originally published in:

Biomaterials 99, https://doi.org/10.1016/j.biomaterials.2016.04.036 


\title{
Novel enzymatically cross-linked hyaluronan hydrogels support the formation of 3D neuronal networks
}

Nicolas Broguiere, Luca Isenmann, Marcy Zenobi-Wong* marcy.zenobi@hest.ethz.ch

\section{Keywords}

Hyaluronan; Hydrogel; Transglutaminase; Neuron; Tissue Engineering;

\begin{abstract}
Hyaluronan (HA) is an essential component of the central nervous system's extracellular matrix and its high molecular weight (MW) form has anti-inflammatory and anti-fibrotic properties relevant for regenerative medicine. Here, we introduce a new hydrogel based on high MW HA which is cross-linked using the transglutaminase (TG) activity of the activated blood coagulation factor XIII (FXIIIa). These HATG gels have significant advantages for neural tissue engineering compared to previous HA gels. Due to their chemical inertness in the absence of FXIIla, the material can be stored long-term, is stable in solution, and shows no cytotoxicity. The gelation is completely cell-friendly due to the specificity of the enzyme and the gelation rate can be tuned from seconds to hours at physiological pH and independently of stiffness. The gels are injectable, and attach covalently to fibrinogen and fibrin, two common bioactive components in in vitro tissue engineering, as well as proteins present in vivo, allowing the gels to covalently bind to brain or spinal cord defects. These optimal chemical and bioactive properties of HA-TG gels enabled the formation of 3D neuronal cultures of unprecedented performance, showing fast neurite outgrowth, axonal and dendritic speciation, strong synaptic connectivity in 3D networks, and rapidlyoccurring and long-lasting coordinated electrical activity.
\end{abstract}

\section{Introduction}

Hyaluronan (HA) is the backbone of the brain and spinal cord extracellular matrix (ECM) [1]. Its binding to proteoglycans, that in turn bind to tenascins, is the ground for the structural integrity of the central nervous system (CNS). Furthermore, the ECM serves as a template for morphogen and growth factor presentation, and variations in its composition are recognized by specialized cell receptors, thereby providing guidance during neural development, plasticity, and regeneration [2]. HA in particular is known to regulate inflammation, with low molecular weight (MW) fragments being pro-inflammatory and high MW chains being anti-inflammatory [3]. High MW HA even limits the glial scarring after brain damage [4] and spinal cord injury (SCI) [5]. It is also an important component for maintaining neural stem cells in vitro and in vivo [6].

Given these advantages, it is no surprise that HA has become a popular tool for neural tissue engineering, with many in vitro and in vivo beneficial effects being reported over the last decade [7]. Standard methods to form HA gels are thiolated HA (HA-SH) cross-linked with acrylated PEG by Michael addition [8], [9], methacrylated HA (HA-MA) photo-crosslinked with a radical initiator [10], and adipic dihydrazide (ADH) cross-linked HA (HA-ADH) [7]. Despite their merits, these cross-linking schemes have major drawbacks which have limited their application. HA gels formed by Michael addition are injectable systems. At physiological pH, however, they gel slowly, making their handling difficult and their clinical 
translation unlikely. For example, investigators needed $25 \mathrm{~min}$ of pre-reaction before injecting such gels into the brain [9]. At high $\mathrm{pH}$, where the reaction proceeds quickly, cell viability is affected. Thiolated HA also readily oxidizes to disulfides, with significant loss in a matter of hours when in solution at physiological $\mathrm{pH}$. In our experience, this becomes particularly problematic with high MW HA-SH (more than $1 \mathrm{MDa}$ ), as it tends to get oxidized to the extent that it forms an insoluble sponge, even when stored frozen or in dry form. This has prompted most users of HA-SH to work with low MW, typically around $100 \mathrm{kDa}$ [11]. Conversely, photo-cross-linking of HA-MA with a radical initiator has the advantage of being based on reagents with higher stability and very fast gelation, but the need for light exposure means such gels are not injectable. Additionally, free radicals are very detrimental to cell viability. It was for example found that neural progenitor cells survive no more than $90 \mathrm{~s}$ during UV exposure in the presence of Irgacure 2959 [10], which prompted the addition of a pre-cross-linking step before mixing in the cells for encapsulation. Finally, ADH cross-linking of HA can only be done in harsh conditions not compatible with neuron encapsulation or in situ gelling $(\mathrm{pH} 3.5-5$ and carboxylic acid activation with a carbodiimide). This makes this method incompatible with in situ gelation and/or cell encapsulation. On an additional note, non-covalent interactions have been investigated in recent years in order to obtain HA formulations with easier handling and improved biocompatibility, e.g. for osteochondral applications [12]. There is no data to our knowledge about the ability of such non-covalent HA gels to provide the very soft yet long-term stable matrices that are needed to sustain neurons.

Overcoming the limitations of these formulations, we present for the first time an HA gel which crosslinks using the specific transglutaminase (TG) activity of the activated blood coagulation factor XIII (FXIIla). This new derivative (HA-TG) is injectable and has a gelling speed tunable from seconds to hours depending on the amount of enzyme added, which makes it very attractive for demanding applications where bleeding and movement may occur. Due to the specific recognition of FXIIla substrate peptides by the enzyme, the cross-linking is completely free of toxic chemicals. FXIIIa is also capable of covalently cross-linking fibrin, its native function, which gives the possibility to co-cross-link such HA derivatives with fibrin(ogen), and also ensures good tissue adhesion. Furthermore, the modified HA is chemically inert, as it is modified with peptides that are not susceptible to oxidation, hydrolysis, or intramolecular Michael addition, unlike thiol or (meth)acrylate derivatives. This guarantees very high stability (no change is visible on the rheological measurements after several months in solution at physiological $\mathrm{pH}$ or after more than one year in frozen dry form). Finally, it is possible to work directly with high MW HA (1-2 $\mathrm{MDa}$ ) without risk of spontaneous cross-linking in storage. This cross-linking method was discovered more than 30 years back [13] and used recently to cross-link PEG gels [14] used in bone tissue engineering applications [15]. Enzymatically cross-linked hydrogels have been extensively applied for drug delivery and cell encapsulation, though the most widespread enzymatic systems have lower bioorthogonality (e.g. HRP-tyramine requires hydrogen peroxide, lysyl oxidase creates aldehydes, and most transglutaminase work uses less specific and more immunogenic bacterial transglutaminases) [16]. Thanks to this new use of FXIIla chemistry, we demonstrate for the first time HA gels encapsulated neurons which were highly viable, quickly extended neurites in 3D, specified axons and dendrites, formed active synapses, and showed long-term stable and coordinated spiking activity.

\section{Materials and methods}


$400 \mathrm{mg}$ ( $1 \mathrm{mmol}$ of the disaccharide repeat unit) of HA sodium salt (Lifecore Biomedical, 1.01-1.8 MDa), and $23.8 \mathrm{mg}(0.1 \mathrm{mmol})$ of 3,3'-Dithiobis(propanoic dihydrazide) (Frontier Scientific) were dissolved slowly in a $150 \mathrm{mM}$ solution of MES hydrate. The resulting $\mathrm{pH}$ was 4.1. When the solution was homogeneous, $38.4 \mathrm{mg}(0.2 \mathrm{mmol})$ of 1-Ethyl-3-(3-dimethylaminopropyl)carbodiimide (EDC, Fluka) were dissolved in $1 \mathrm{ml}$ of $\mathrm{mQ}$ water and added dropwise with stirring. The solution was quickly homogenized, and then stirring was stopped and the reaction left to react for at least $4 \mathrm{~h}$ (to completion). The $\mathrm{pH}$ increases towards $\sim 4.5$ during the course of the reaction, with MES buffer preventing it from going higher. Then, $143.33 \mathrm{mg}(0.5 \mathrm{mmol})$ of TCEP-HCl (Fluorochem) was added from $500 \mu \mathrm{l}$ water, the solution was homogenized by swirling a few times, and the reduction was left to proceed overnight in a standing sealed flask. Finally, $1 \mathrm{~g}(17 \mathrm{mmol})$ of $\mathrm{NaCl}$ was added to the solution, and the mix was dialyzed against $\mathrm{mQ}$ water brought to $\mathrm{pH} 4.5$ with dilute $\mathrm{HCl}$, with 4 water changes over $24 \mathrm{~h}$, to yield a solution of pure HA-SH, sodium salt. Thiolation rate was quantified during preliminary optimization with Ellman's test in phosphate buffer $\mathrm{pH} 7.27$ using a beta-mercaptoethanol calibration curve. The hydrazide conjugation rate was found to be $\sim 50 \%$. For subsequent batches, quantifications were done only at the next step, which is more precise because VS give distinct peaks on proton NMR that can be compared to an internal standard ( $\mathrm{N}$-acetylate from $\mathrm{HA}$ backbone) to avoid errors due to impurities (e.g. bound water) in the starting materials.

Thiols oxidize rapidly, yielding HA gels (in solution) or insoluble sponges (in dry or frozen forms), particularly if the solution is neutralized or stored in the presence of oxygen. Hence, for best reproducibility, we proceeded directly with the next step.

\section{HA-VS synthesis}

The HA-SH solution recovered from the previous dialysis was added dropwise into a solution of $1 \mathrm{ml}$ (10 $\mathrm{mmol}$ ) divinyl sulfone (DVS, Sigma) in $40 \mathrm{ml}$ of TEOA buffer, $300 \mathrm{mM}, \mathrm{pH} 8.0$. The reaction was left to proceed for $2 \mathrm{~h}$ at $\mathrm{RT}$, and then $1 \mathrm{~g}$ of $\mathrm{NaCl}(17 \mathrm{mmol})$ was added and the solution was dialyzed against $\mathrm{mQ}$ water to yield pure vinyl sulfone-substituted HA (HA-VS).

The substitution rate was measured by comparing the peaks at $6.75 \mathrm{ppm}$ (vinyl sulfones) and $1.75 \mathrm{ppm}$ ( $\mathrm{N}$-acetyls from HA) from proton NMR and found to be of $10 \%$ of the repeat unit disaccharide carboxylic acids (Fig S1).

HA-VS is stable for extended periods of time in solution (at least weeks), but hardly resuspends if frozen or lyophilized, probably due to hydrophobic interactions. We therefore proceeded directly with the next step (if storage in dry form is necessary, lyophilizing in the presence of $>20 \mathrm{mM} \mathrm{NaCl}$ yields a powder that can be easily resuspended).

\section{HA-TG/Lys and HA-TG/GIn}

The HA-VS solution recovered from the previous dialysis was split in two equal parts. One half was substituted with a transglutaminase substrate peptide that provides a reactive glutamine residue (TG/GIn: NQEQVSPL-ERCG) and the other half a lysine residue (TG/Lys: FKGG-GPQGIWGQ-ERCG). The middle sequence imparts MMP sensitivity, so gels can be slowly biodegraded (or simply trypsinized for cell recovery). Peptides were from Anawa, and contained $\sim 40 \%$ salts. The expected molarity of vinyl 
sulfone groups in each half was $50 \mu \mathrm{mol}$, so we added $65 \mu \mathrm{mol}$ in each case, to provide a slight excess of peptide. $10 \mathrm{ml}$ of TEOA buffer $300 \mathrm{mM}$, pH 8.0 was added to each half of the HA-VS solution, the mix were deoxygenated by bubbling with nitrogen gas, and finally the peptides were added. The solutions were quickly homogenized, the flasks sealed, and the reactions left to proceed overnight without stirring. Finally, $2 \mathrm{~g}$ of $\mathrm{NaCl}$ were dissolved in each flask, and the product was dialyzed against mQ water. The resulting pure HA-TG components were sterilized by filtration, aliquoted and lyophilized in sterile conditions. Complete substitution was confirmed by disappearance of the VS peaks on proton NMR.

\section{FXIII activation}

FXIII (Fibrogammin, CSL Behring) was resuspended at $200 \mathrm{U} / \mathrm{ml}$ in $\mathrm{mQ}$ water, aliquoted by $100 \mathrm{ul}$ and stored at $-80^{\circ} \mathrm{C}$. For activation, $3.2 \mu \mathrm{l}$ of thrombin (from Tissucol, Baxter) at $50 \mathrm{U} / \mathrm{ml}$ and $2 \mu \mathrm{l}$ of $100 \mathrm{mM}$ $\mathrm{CaCl} 2$ were added to one aliquot, and incubated for $15 \mathrm{~min}$ at $37^{\circ} \mathrm{C}$. Activated FXIII (FXIIla) was stored as $10 \mu$ aliquots at $-80^{\circ} \mathrm{C}$ until the day of use.

\section{HA-TG gel formation}

Aliquots of HA-TG/Lys and HA-TG/GIn were resuspended at the desired concentration, i.e. $0.5 \%(\mathrm{w} / \mathrm{v})$ unless indicated otherwise, in saline supplemented with $50 \mathrm{mM}$ TRIS and $50 \mathrm{mM} \mathrm{CaCl}$, with $\mathrm{pH}$ balanced to 7.6 and sterilized by filtration (referred to as TBS). Then, the two solutions were combined in equal volume, and the amount of enzyme corresponding to the desired gelling speed was added to the wall of the tube, i.e. $3 \mu \mathrm{l}$ of FXIIla $200 \mathrm{U} / \mathrm{ml}$ for $80 \mu \mathrm{l}$ of gel unless indicated otherwise, and incorporated quickly by vortexing. Gelation occurred within 1-2 minutes, and was left to proceed for 20 minutes to ensure a stiffness plateau is reached before transferring to medium.

\section{Rheometry}

After adding the FXIIla, the gel precursor was quickly loaded on an Anton Paar MCR 301 rheometer equipped with a $20 \mathrm{~mm}$ plate-plate geometry and metal floor, pre-warmed to $37^{\circ} \mathrm{C}$ and with humidified chamber. The probe was quickly lowered to measuring position (100 to $200 \mu \mathrm{m}$, monitoring the gel precursor forming a crown around the geometry while lowering the probe to ensure the measuring space is precisely filled). Then the gelling was monitored at $1 \mathrm{~Hz}$ with $4 \%$ strain.

Control fibrin gels were made by adding FXIIla and thrombin at 7.5 and $0.5 \mathrm{U} / \mathrm{ml}$ respectively from concentrated stocks into a $0.5 \%(\mathrm{w} / \mathrm{v})$ fibrinogen solution in TBS. HA-Fibrin hybrids were made by adding the same amount of enzyme in a fibrinogen solution additionally containing $0.5 \%$ (w/v) HA-TG.

For measurements after swelling (Fig. S2A), 0.5\% (w/v) HA-TG gels were made in $1.4 \mathrm{~mm}$ thick Teflon casters, left to gel for 20 min at $37^{\circ} \mathrm{C}$ with a humidified atmosphere, collected and submerged in PBS pH7.4 for 3 days. The gels were then loaded on the rheometer equipped with a $10 \mathrm{~mm}$ plate-plate geometry, compressed by $10 \%$ to ensure surface adhesion, trimmed, and measured with a frequency sweep at $4 \%$ strain.

\section{Neuron cultures}


Cortices from E17 Wistar rat embryos were dissected and dissociated as described previously [17]. Briefly, the cortices were incubated for $15 \mathrm{~min}$ at $37^{\circ} \mathrm{C}$ in PBS supplemented with $1 \mathrm{mg} / \mathrm{ml} \mathrm{BSA}, 10 \mathrm{mM}$ glucose, $0.5 \mu \mathrm{g} / \mathrm{ml}$ DNAse (Sigma, D-5025) and $0.5 \mathrm{mg} / \mathrm{ml}$ papain (Sigma, P-4762), and washed with blocking medium consisting of DMEM $+10 \%$ FBS. They were resuspended in $2 \mathrm{ml}$ of blocking medium, and dissociated by trituration using a fire polished Pasteur pipette. The dissociated cells were resuspended in serum free growth medium, consisting of Neurobasal + B27 (Gibco 21103-049 and 17504-044) supplemented with 1x Glutamax and Penicillin/Streptomycin, and plated overnight on polyL-lysine coated flasks ( $50 \mathrm{ug} / \mathrm{ml}$ in borate buffer, $\mathrm{pH} 8.4$, incubated at $37^{\circ} \mathrm{C}$ for $1 \mathrm{~h}$ ). The following day, the plated neurons were first washed with TrypLE express for $5 \mathrm{~min}$, to detach weakly adherent non-viable cells and cell debris. The remaining healthy cells were then detached with trypsin/EDTA (Gibco 12605010 and 25200-072). After addition of two volumes of blocking medium, the cells were centrifuged and resuspended in serum-free growth medium, and counted.

For encapsulation, the neurons were pelleted and resuspended at $10 \mathrm{e} 6 \mathrm{cells} / \mathrm{ml}$ in the HA-TG/Lys-Gln mix. After addition of the FXIIla, the gel precursor was cast into home-made $4 \mathrm{~mm}$ diameter $/ 1 \mathrm{~mm}$ height PDMS molds pre-adhered onto coverslips, and kept in 24 well plates. After 20 minutes of gelling at $37^{\circ} \mathrm{C}$ in a humidified atmosphere, $1 \mathrm{ml}$ of serum-free medium was added onto the gels. After 2-3 weeks, half of the medium was replaced once a week.

\section{Live-dead assays}

Gels were incubated $1 \mathrm{~h}$ in medium supplemented with calcein AM $2 \mu \mathrm{M}$ and propidium iodide 6.6 $\mu \mathrm{g} / \mathrm{ml}$, transferred to fresh medium for washing and imaged on a Leica SP8 multiphoton over $465 \times 465 \times 200 \mu \mathrm{m}$. Live and dead cells were counted manually on the maximum intensity projection (MIP) (typically $\sim 400$ cells/image). Experiments were reproduced in triplicate with 3 different litters $(n=9)$.

\section{Immunocytochemistry and imaging}

Primary antibodies were: $\beta$ III-tubulin (Sigma T5076, 1:500), neurofilament (Sigma N4142, 1:150), synaptotagmin (DSHB, $8 \mu \mathrm{g} / \mathrm{ml}$ ), MAP-2 (Sigma M3696, 1:200), PSD-95 (Abcam ab18258, 1:400). Secondary antibodies conjugated with Alexa 488 and 594 (Invitrogen A11005, A11008, A10680, A11015) were used at 1:200.

We adapted standard immunocytochemistry protocols to stain and image directly the entire gels. Samples were fixed and permeabilized for $1 \mathrm{~h}$ at $4^{\circ} \mathrm{C}$ in $10 \%$ formalin with $0.1 \%$ Triton X-100, blocked overnight with $5 \%$ BSA in PBS, and washed with PBS (twice $1 \mathrm{~h}$, once overnight). They were then incubated in primary antibody (overnight at RT with gentle shaking, dilution with $3 \%$ BSA in PBS), washed with PBS, incubated overnight in secondary antibody, washed with PBS, stained with DAPI $0.3 \mu \mathrm{M}$ in PBS for $1 \mathrm{~h}$, and finally washed with PBS.

Imaging of immunostained samples was performed on a Leica SP8 multiphoton microscope with a 20x/0.95 NA water objective, typically exciting at $710 \mathrm{~nm}$ and $1100 \mathrm{~nm}$ using MaiTai Deepsee and Insight Deepsee fs-lasers respectively (Spectra Physics). The resulting stacks were edited in Fiji to apply a MIP, and adjust the color display (brightness/contrast/gamma). When salt and pepper noise was present, median filtering over $<2$ px was applied. 


\section{Spiking activity imaging}

The genetically encoded intracellular calcium reporter GCaMP was delivered with an adeno-associated virus added directly into the gel precursor at optimized concentration. The spiking activity was then imaged either on a Leica SP8 confocal microscope at 8 frames/s (Fig. 5) or on a Zeiss observer widefield microscope with $\mathrm{CO} 2$ incubation at 2.5 frames/s (Fig. 6).

To confirm that calcium waves were indeed associated with neuronal electrical spikes and glutamatergic transmission, the following selective inhibitors were added in the medium and left to diffuse into the gels for $1 \mathrm{~h}$ : Tetrodotoxin $500 \mathrm{nM}$ (Acros Organics, voltage gated sodium channel antagonist), CPP $10 \mu \mathrm{M}$ (Sigma, NMDA glutamate receptor antagonist), CNQX $20 \mu \mathrm{M}$ (Sigma, AMPA/kainate glutamate receptor antagonist).

\section{Results and discussion}

\section{Synthesis of HA-TG gels}

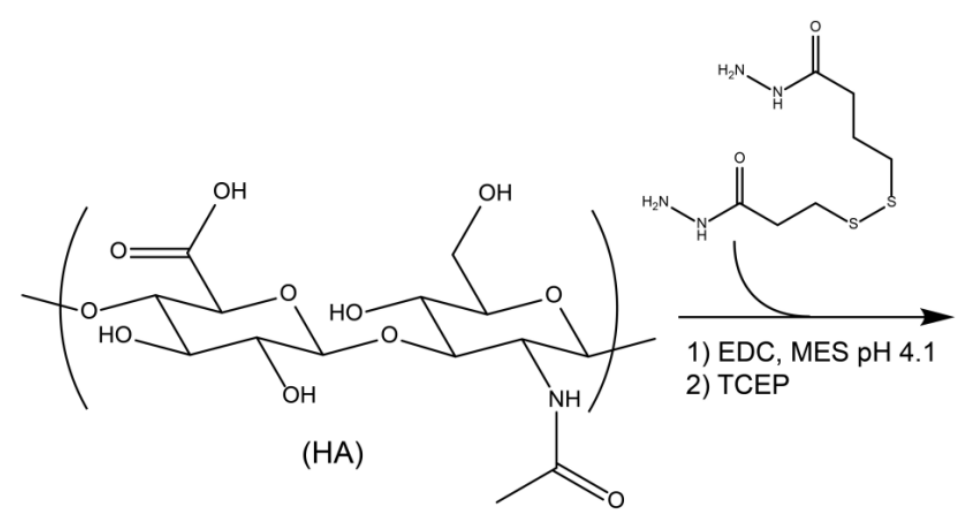

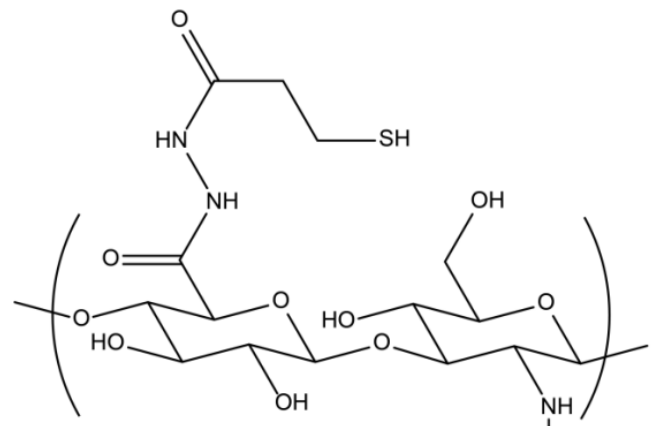

(HA)

(HA-SH)
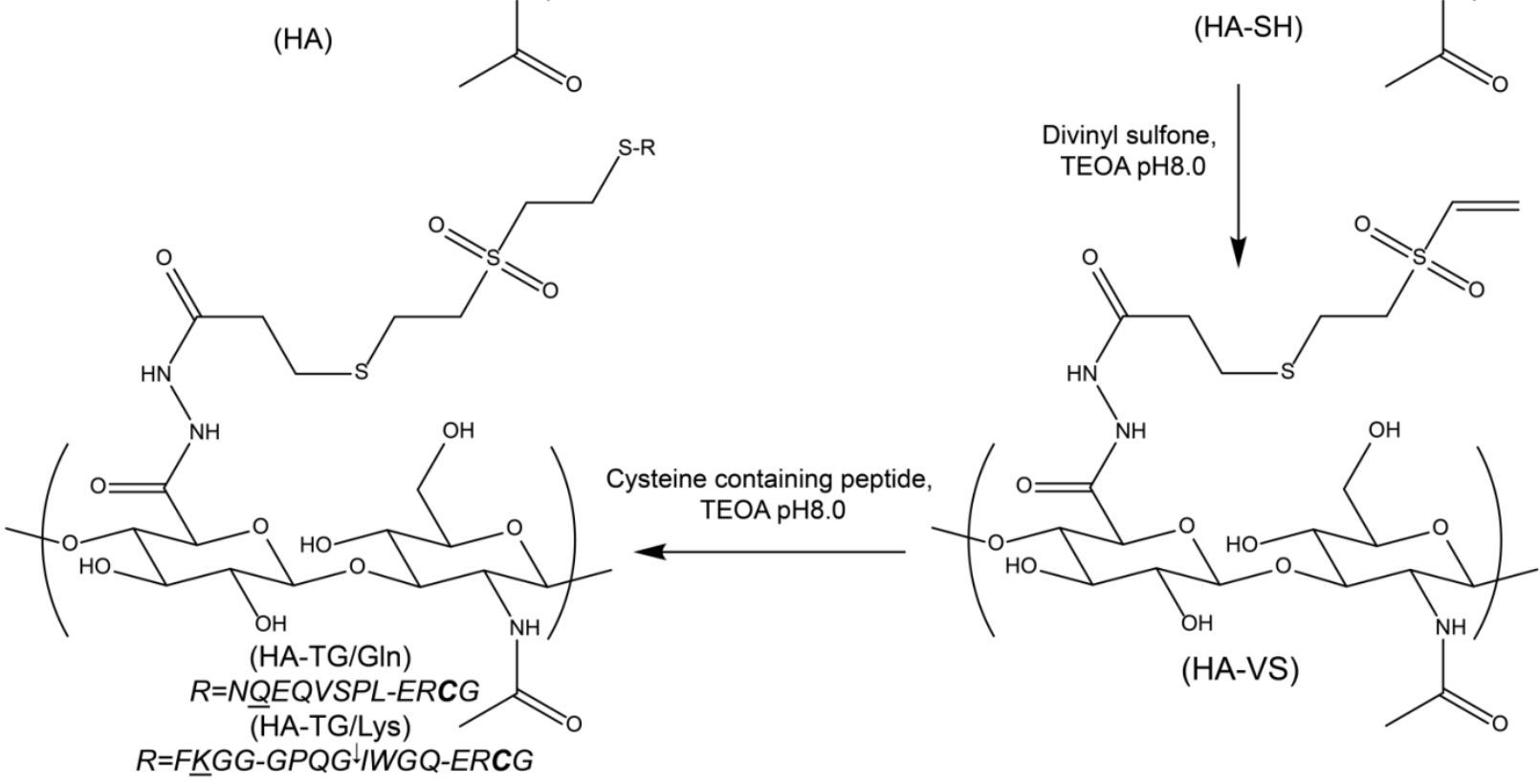

Figure 1. Synthesis of the HA derivatives. In the TG peptides, the cysteines that provide thiols for conjugation onto HA-VS are in bold and the lysine and glutamine covalently coupled to each other on their side chains by FXIIla are underlined. The MMP cleavage site is marked by an arrow. 
High MW HA (which is a linear, highly water soluble polysaccharide) was modified with peptides in order to render it covalently cross-linkable by the enzymatic activity of FXIlla. Solutions of the polymer precursors HA-TG/Lys and HA-TG/GIn are stable and inert in buffer solution, but in the presence of FXIIla the side chains of the reactive lysine and glutamine residues are cross-linked to each other with a new amide bond, which results in hydrogel formation.

The synthesis of the HA-TG precursor polymer was done in three steps (Fig. 1): first, HA was derived with thiols using EDC activation and hydrazide conjugation. This is the critical step that defines the rate of substitution and therefore the subsequent gelation behavior and mechanical properties of the gel. Then, the very simple and efficient Michael addition of thiols on vinyl sulfones is used to substitute these thiols with vinyl sulfones, and finally with the peptides that FXIIIa can ligate, namely TG/lys and TG/GIn.

Hydrogels for neuron use should ideally be very soft (no more than a few hundred Pa) and with large pore size [18]. This is most naturally achieved by gelling high MW polymer with low cross-link density. Therefore, we used HA of 1-2 MDa as starting product, and a great deal of attention was paid to avoid any unnecessary stirring, $\mathrm{pH}$ balancing, lyophilizing, precipitating, or filtering step, as all of those processes are known to be hard to perform on viscous solutions and/or causing chain breakage and MW loss. Therefore, buffers were used to keep the $\mathrm{pH}$ within the desired ranges without tracking at every step. Dialysis is very efficient with such high MW polymers and the most gentle purification technique, and was therefore used systematically.

This 3-step process is preferred over direct conjugation of peptides onto the native HA because carbodiimide activated conjugations of unprotected peptides on polysaccharides in aqueous conditions have poor specificity (side chain amines can react, even if terminal amines have higher reactivity), poor efficiency (which is a problem as peptide are expensive reagents), and poor reproducibility in terms of rate of substitution (for example because the exact salt and TFA contents in purified peptides are highly variable). It is also preferable to first substitute with thiols rather than directly with DVS, because the degree of substitution of DVS on HA hydroxyls would have to be time and $\mathrm{pH}$ controlled, which is very sensitive and therefore not optimal for reproducibility. Also, in our hands, direct substitution with DVS on HA hydroxyls [19] works reasonably well for low molecular weight HA only: with a molecular weight of 1-2 MDa, interchain cross-links resulting in gel or precipitate formation are almost unavoidable. Even for low MW HA, the degree of substitution is critically sensitive to small variations of $\mathrm{pH}$ and reaction time, which is better to avoid in order to improve the reproducibility. On the other hand, the substitution with protected thiols through hydrazide addition on activated carboxylic acids is simply pushed to reaction completion. This ensures that the degree of substitution is easily and precisely reproducible (as confirmed by rheometry and NMR), as long as the weight of the reagents added is measured precisely.

A major motivation for choosing to move on from HA-SH and HA-VS to HA-TG was that thiols get readily oxidized, and very little oxidation was in our experience enough to form a cross-linked sponge when working with very high MW HA. This complicates long-term storage of $\mathrm{HA}-\mathrm{SH}$, especially if neutralized. It also negatively affects reproducibility, as solutions of high $\mathrm{MW} \mathrm{HA-SH}$ in buffer at $\mathrm{pH} 7.4$ undergo significant oxidation even during the few hours of a single experiment. High MW HA-VS, despite of its relatively good stability in solution, has similar storage issues because it hardly resuspends from frozen or lyophilized form, probably because of quickly occurring hydrophobic interactions as well as slow 


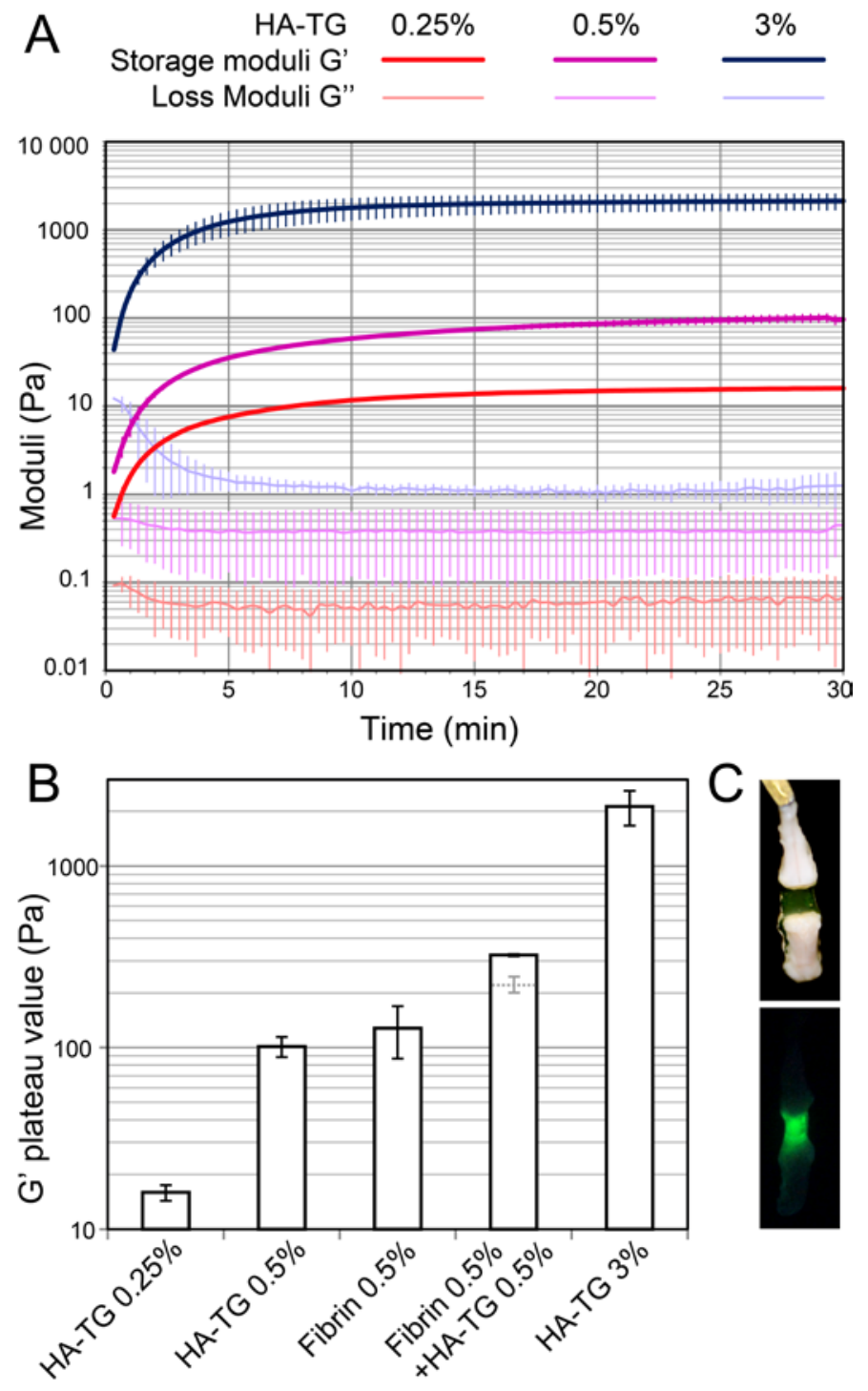

occurring additions of HA hydroxyls or deacetylated amines on the vinyls while in the solid state. HA-TG derivatives had perfect stability in solution, in frozen, and in dry form, as checked by rheometry (no change in the gelling curve was found after the polymer had spent several months in TBS buffer $\mathrm{pH}$ 7.6 at $4^{\circ} \mathrm{C}$, or more than a year in dry frozen form, Fig. S2C-D).

Figure 2. Mechanical measurements. (A) Gelling profiles for different concentrations of HA-TG as monitored by rheometry and (B) Values of the storage modulus after $30 \mathrm{~min}$ (plateau), showing fibrin gels and HA-fibrin hybrids. The dashed line on the HA-fibrin hybrid gel shows the expected modulus if there would be no co-cross-linking or interactions between the $H A$ and fibrin networks. Enzyme concentration was adjusted to keep similar gelation speed for all conditions, respectively in U/ml: FXIIla 10 for HA-TG 0.25\%, FXIIIa 7.5 for HA-TG 0.5\%, FXIII 20 and thrombin 12.5 for HA-TG 3\%, FXIIIa 7.5 and thrombin 0.5 for Fibrin and Fibrin+HA-TG. Error bars: SD from 3 to 4 replicates. (C) Demonstration of gel adhesion to mouse spinal cord tissue ex vivo. The tissue is

hanging from a forceps visible on top and the middle fluorescent segment is the gel.

Nevertheless, the advantages of HA-TG go far beyond stability in storage. First of all, control of the gelling speed with the dose of enzyme enables very quick gelling (Fig. S2B), overcoming a major hurdle associated with the Michael addition. As seen on Fig. 2A, gelling can be adjusted to happen within the first minute after sample mixing and delivery. Human FXIIla is available in large quantity and clinical quality, as an injectable drug. Stiffness from 10 to $2000 \mathrm{~Pa}$ is easily accessible with variations of the HATG concentration, and the gelling speed can be dissociated from the final stiffness at physiological $\mathrm{pH}$.

Monitoring in weight during free swelling in PBS showed no change in the mass of the gels (so no change in their volume, since their density is always that of water). After washing with $\mathrm{mQ}$ and drying, the same mass of polymer as that which was introduced initially was found, showing the polymer is fully crosslinked and the gel stable.

As FXIIla's native function is to cross-link fibrin clots with themselves and with associated proteins, it is expected that copolymers of fibrin and HA-TG can be formed, as shown in Fig. 2B. The stiffness of a gel 
containing fibrin and HA is higher than the sum of the stiffness of the fibrin or HA gels alone, showing synergistic interactions are occurring. HA-TG gels were also found to adhere to mouse spinal cord tissue, which is an important property for the envisioned future in vivo applications (Fig 2C).

Neuron encapsulation: viability and morphology
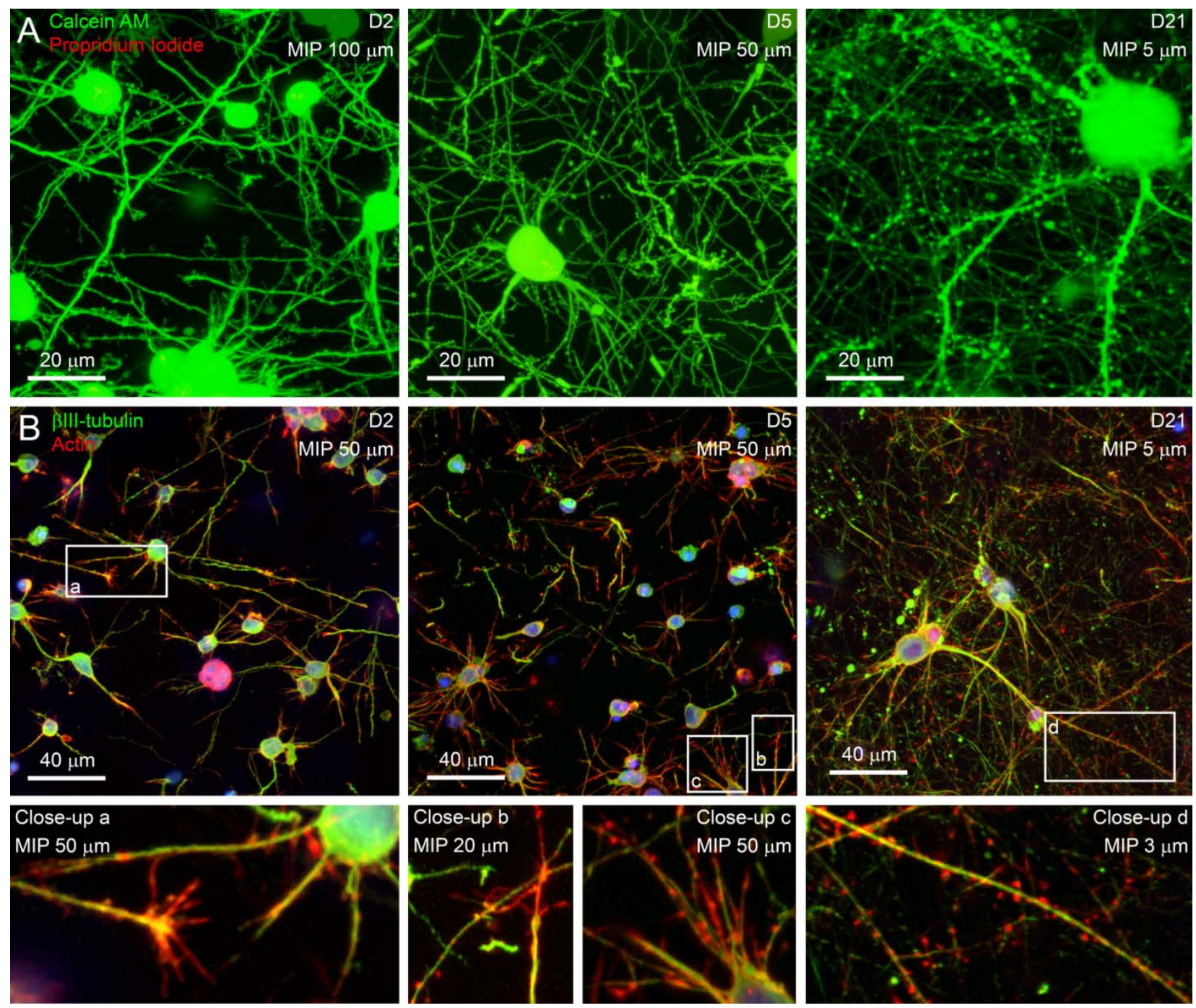

Figure 3. Neuron morphology (A) as seen with calcein staining of all the intracellular space and propidium iodide staining of dead cell nuclei and (B) as seen with cytoskeletal staining. D2, D5, and D21 refer to the number of days neurons spent after encapsulation in the gels. Note the tremendous increase in neurite density over time as maximum intensity projections (MIP) are done over reduced thickness at later time points. Close-ups emphasize axonal and dendritic growth cones, as well as actin-filled synaptic buttons budding from microtubule-filled dendrites at later time points.

Being inert in the absence of FXIIla also had the consequence that HA-TG reagents had no cytotoxicity whatsoever, and dissociated neurons could be encapsulated without loss of viability. Pilot experiments found that $0.25 \%$ gels tended to be too soft to be easily handled without damage, whereas $1 \%$ gels had reduced neurite outgrowth. We therefore conducted the study with $0.5 \%$ HA-TG (half of it being HA- 
TG/Lys, and half of it HA-TG/GIn). These gels did not appear to degrade, nor did they swell or shrink noticeably over culture periods of typically 1 to 2 months. Under these conditions, the viability was around $90 \%$ just before encapsulation (D0), and found to be of $85 \pm 5 \%$ at D2 and $81 \pm 7 \%$ at D5 (SD n=9). The gels showed no sign of degradation at any time point and stable cultures could be kept for more than 2 months.

Fast neurite extension was seen from the first days, and by D21, the gels were filled by a dense mesh of neurites, forming extensive 3D networks (note the reduced MIP thickness and increased neurite densities in Fig. 3A from D2 to D21). Large growth cones with many exploratory actin filled filipodia at the tip of microtubule-filled axons, as well as smaller and simpler dendritic growth cones, were reminiscent of what is seen in vivo (Fig. 3B close-ups $a-b$ ). Neurites were also covered with many actinfilled small filaments at D2, that might be branching points initiations. By D5, they appeared mostly smooth. Afterwards, actin-filled buds reminiscent of dendritic spines started to be visible on some neurites at D5, and became omnipresent by D21 (clearly visible in Fig. 3A D2-D21 and Fig. 3B close-ups bc-d).
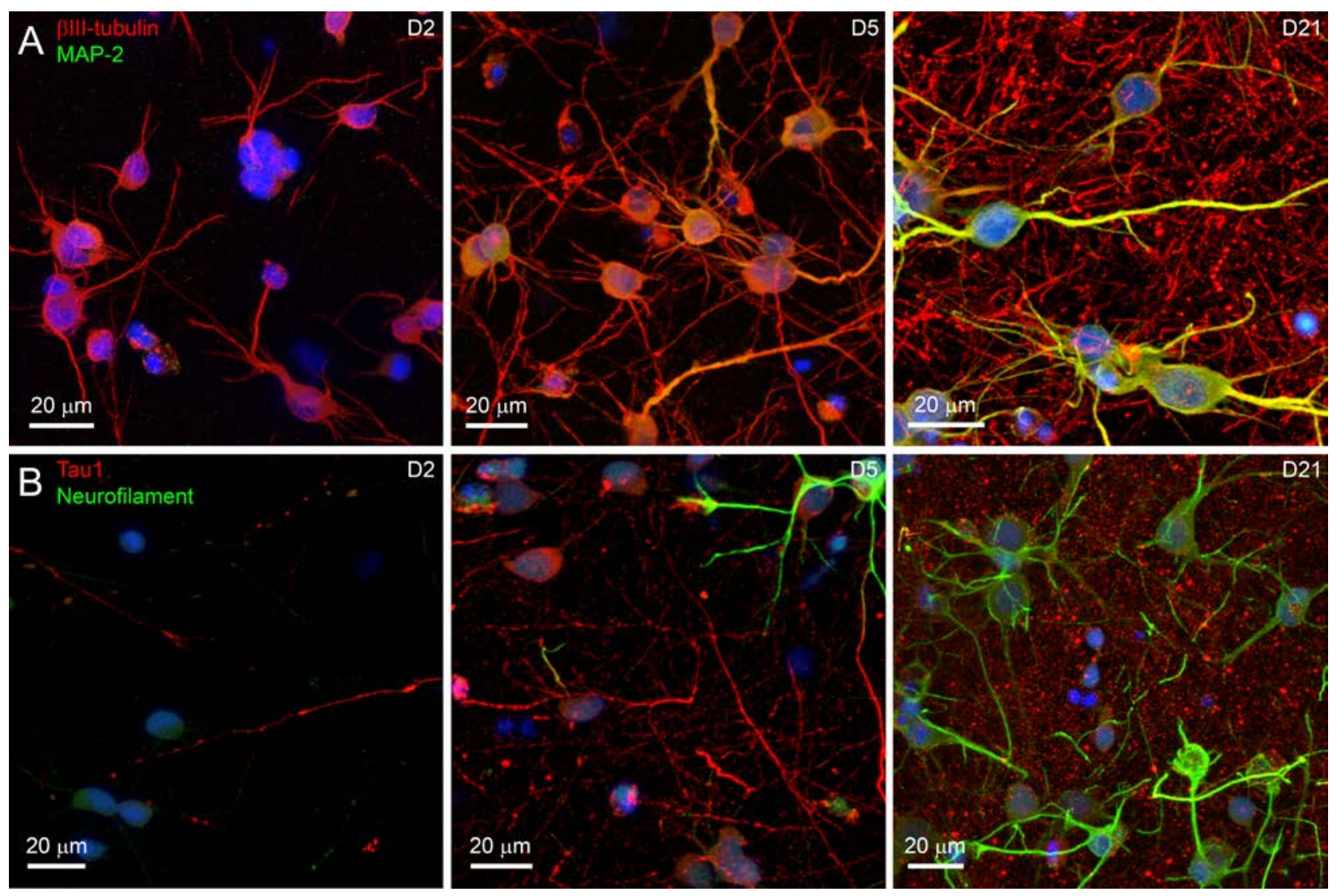

Figure 4. Axonal and dendritic speciation. (A)The mature dendrite marker MAP-2 starts to be expressed in the cell bodies and proximal neurites at D5, and becomes strongly expressed in the whole length of a subset of neurites at D21. The embryonic neuron marker Bll-tubulin is present in all neurites. (B) The axonal marker Tau1 is already segregated in some neurites that are therefore axons at D2. By D5, many axons span the gel, and by D21 too many Tau1-positive debris are scattered through the gel to distinguish axons. The mature neuron marker neurofilament starts to be expressed strongly in a few 
neurons at D5 and is strongly present in a subset of neurites of most neurons at D21 (all neurons have some weak staining at all time points). All pictures are $50 \mu \mathrm{m}$ MIP.

Specific axonal and dendritic markers appeared at the same time as the morphological characteristics associated with axons and dendrites: Tau1 positive axons were already visible at D2, and the dendritic marker MAP-2 started to be expressed in the cell bodies and proximal dendrites at D5, and became strongly expressed and localized to the whole length of a subset of neurites by D21 (Fig. 4). $\beta$ III tubulin stains all neurites from these embryonic neurons, as expected, while neurofilaments were strongly expressed only after 21 days, and only in a subset of neurites.

\section{Spiking activity}
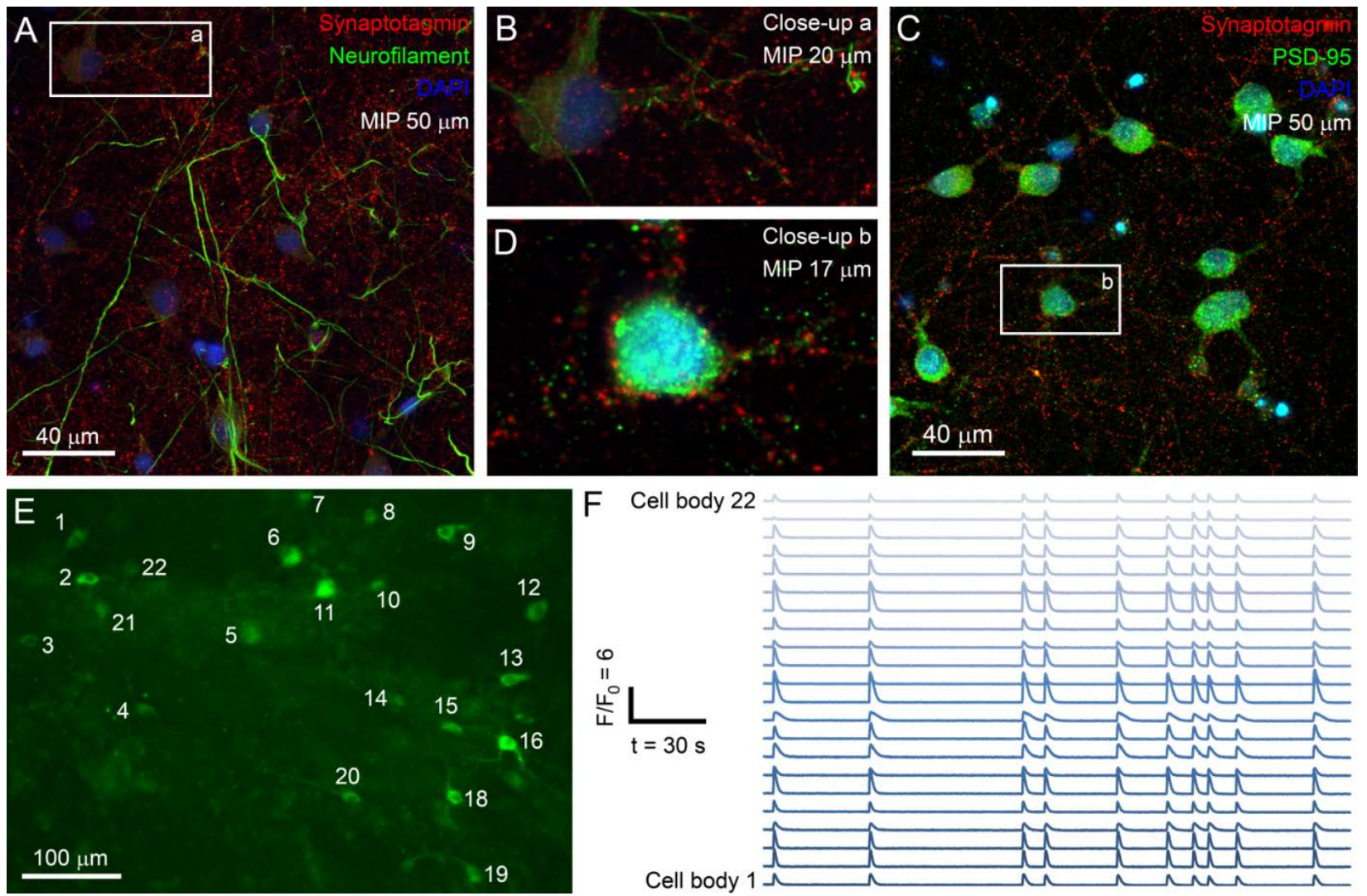

Figure 5. Synapse formation and coordinated spiking activity. (A) There is a dense mesh of potential presynaptic densities marked by Synaptotagmin (B) the synaptic densities are densely packed on the surface of cell bodies and dendrites (C) There is a similarly dense mesh of potential post-synaptic densities marked by PSD-95 (D) The pre and post synaptic densities are often found in close apposition to each other, showing many synapses are present. (E) Neurons transfected with the genetically encoded calcium reporter GCaMP (F) Measurement of calcium level in the neurons of (E) with high speed confocal microscopy. All neurons in the field of view spike fully synchronously, confirming strong synaptic connectivity. $(A-D)$ are at $D 21$ and $(E-F)$ at D10.

The genetically encoded calcium reporter GCaMP was used to monitor spiking activity of the neurons. Strong and fully synchronous spiking was already visible at D10 (Fig. 5 E-F). In agreement with this, a 
great density of synaptotagmin-filled potential presynaptic terminals and PSD-95 filled potential postsynaptic terminals were found associated with cell bodies and neurites (Fig. 5 A-B) and in many cases associated with each other (C-D), to form synapses.

As calcium waves can be present in other cell types than neurons and have other causes than electrical spiking, we confirmed the spiking was indeed neuronal electrical activity by blocking with the specific voltage gated sodium channel blocker Tetrodotoxin (TTX). In addition, specific inhibitors of glutamatergic transmission CPP and CNQX blocked the spiking activity as well, proving the neuronal excitation is based on standard glutamatergic excitation, as could be expected from cortical neurons (Fig. 6). Activity was always recovered after washing overnight, showing none of the compounds were used at a toxic dose.

The ease with which neurons in 3D culture in these gels can be monitored with fluorescent reporters together with the fact that pharmacological testing with small molecules can be done by simply letting molecules diffuse $1 \mathrm{~h}$ through the gels makes these gels particularly fitting for neurobiology or pharmacology studies in 3D cultures.

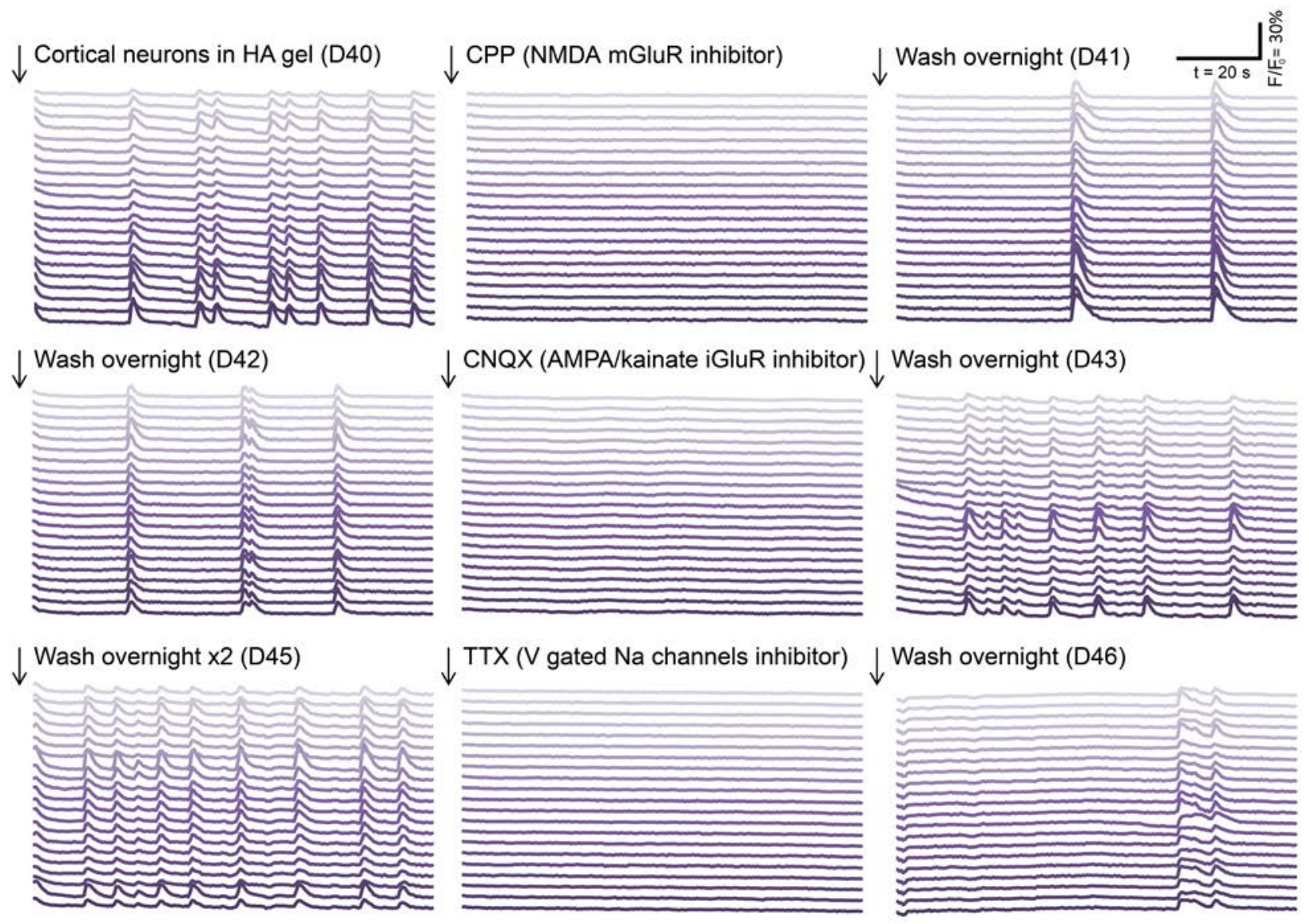

Figure 6. Characterization of the spiking activity with pharmacological blockers confirms that calcium spikes are associated with neuronal electrical activity and glutamatergic excitation. Each line shows the calcium level in a different cell body from fluorescent reporter imaging, and the arrows indicate media changes, with incubations of $30 \mathrm{~min}$ to $1 \mathrm{~h}$ when adding an inhibitor and up to overnight for washing steps. 


\section{Conclusion}

The new HA-TG hydrogels described here cross-link with the specific transglutaminase activity of FXIIIa and present an ideal set of properties for neural tissue engineering, including chemical stability, specific cross-linking with independently tunable gelation speed and stiffness, cytocompatibility with dissociated neuron encapsulation (the most demanding cytocompatibility test), injectability, covalent cross-linking to fibrin and other proteins, and the possibility to enzymatically degrade the gels for bioresorption or cell recovery. These gels are additionally based on high MW HA, which has important neuroprotective, antifibrotic, and anti-inflammatory properties. HA is additionally the backbone of the brain ECM, so neurons are provided with a gel which mimics their natural environment. All these properties enabled 3D neuronal cultures of unprecedented performance, with fast neurite outgrowth, proper neuron polarization, quickly-occurring and long-lasting electrical activity with strong synaptic connectivity. These gels are therefore perfectly positioned to bring neurobiological and pharmacologic cell-based studies to the third dimension. They are promising as well for translation to brain or spinal cord injury applications, something which will require further in vivo investigation.

\section{Acknowledgements}

This work was supported by ETH internal funding. We like to thank Prof. Jean-Marc Fritschy and Giovanna Bosshard, University of Zurich for kindly providing the rat embryo cortices and Prof. Botond Roska, Friedrich Miescher Institute for Biomedical Research, Basel for the AAV- EF1a-GCaMP6 construct. We also acknowledge PennVector for the development of the AAV vector, and J. Juettner and Dr. Palazzolo for teaching virus production protocols, as well as support of the Scientific Center for Optical and Electron Microscopy (ScopeM) of ETH Zurich. The monoclonal antibody mAB 30, developed by Dr. Reichardt from UCSF, was obtained from the Developmental Studies Hybridoma Bank, created by the NICHD of the NIH and maintained at The University of lowa, Department of Biology, lowa City, IA 52242.

\section{References}

[1] E. Ruoslahti, "Brain extracellular matrix," Glycobiology, vol. 6, no. 5, pp. 489-492, 1996.

[2] D. R. Zimmermann and M. T. Dours-Zimmermann, "Extracellular matrix of the central nervous system: from neglect to challenge.," Histochem. Cell Biol., vol. 130, no. 4, pp. 635-53, Oct. 2008.

[3] K. S. Girish and K. Kemparaju, "The magic glue hyaluronan and its eraser hyaluronidase: a biological overview.," Life Sci., vol. 80, no. 21, pp. 1921-43, May 2007.

[4] C. M. Lin, J. W. Lin, Y. C. Chen, H. H. Shen, L. Wei, Y. S. Yeh, Y. H. Chiang, R. Shih, P. L. Chiu, K. S. Hung, L. Y. Yang, and W. T. Chiu, "Hyaluronic acid inhibits the glial scar formation after brain damage with tissue loss in rats," Surg. Neurol., vol. 72, no. SUPPL. 2, pp. S50-S54, 2009.

[5] Z. Z. Khaing, B. D. Milman, J. E. Vanscoy, S. K. Seidlits, R. J. Grill, and C. E. Schmidt, "High molecular weight hyaluronic acid limits astrocyte activation and scar formation after spinal cord injury.," $J$. Neural Eng., vol. 8, no. 4, p. 046033, 2011.

[6] Z. Z. Khaing and S. K. Seidlits, "Hyaluronic acid and neural stem cells : implications for biomaterial design," J. Mater. Chem. B, vol. 3, pp. 7850-7866, 2015. 
[7] X. Wang, J. He, Y. Wang, and F.-Z. Cui, "Hyaluronic acid-based scaffold for central neural tissue engineering," Interface Focus, vol. 2, no. 3, pp. 278-291, 2012.

[8] D. Eng, M. Caplan, M. Preul, and A. Panitch, "Hyaluronan scaffolds: A balance between backbone functionalization and bioactivity," Acta Biomater., vol. 6, no. 7, pp. 2407-2414, 2010.

[9] Y. Liang, P. Walczak, and J. W. M. Bulte, "The survival of engrafted neural stem cells within hyaluronic acid hydrogels," Biomaterials, vol. 34, no. 22, pp. 5521-5529, 2013.

[10] S. K. Seidlits, Z. Z. Khaing, R. R. Petersen, J. D. Nickels, J. E. Vanscoy, J. B. Shear, and C. E. Schmidt, "The effects of hyaluronic acid hydrogels with tunable mechanical properties on neural progenitor cell differentiation.," Biomaterials, vol. 31, no. 14, pp. 3930-40, May 2010.

[11] X. Zheng Shu, Y. Liu, F. S. Palumbo, Y. Luo, and G. D. Prestwich, "In situ crosslinkable hyaluronan hydrogels for tissue engineering," Biomaterials, vol. 25, no. 7-8, pp. 1339-1348, Mar. 2004.

[12] J.-H. Shim, K.-M. Jang, S. K. Hahn, J. Y. Park, H. Jung, K. Oh, K. M. Park, J. Yeom, S. H. Park, S. W. Kim, J. H. Wang, K. Kim, and D.-W. Cho, "Three-dimensional bioprinting of multilayered constructs containing human mesenchymal stromal cells for osteochondral tissue regeneration in the rabbit knee joint," Biofabrication, vol. 8, no. 1, p. 014102, 2016.

[13] A. Ichinose, T. Tarnaki, and N. Aoki, "Factor XIII-mediated cross-linking of NHZ-terminal peptide of az-plasmin inhibitor to fibrin," FEBS Lett., vol. 153, no. 2, pp. 369-371, 1983.

[14] M. Ehrbar, S. C. Rizzi, R. Hlushchuk, V. Djonov, A. H. Zisch, J. a Hubbell, F. E. Weber, and M. P. Lutolf, "Enzymatic formation of modular cell-instructive fibrin analogs for tissue engineering.," Biomaterials, vol. 28, no. 26, pp. 3856-66, Sep. 2007.

[15] U. Blache, S. Metzger, Q. Vallmajo-Martin, I. Martin, V. Djonov, and M. Ehrbar, "Dual Role of Mesenchymal Stem Cells Allows for Microvascularized Bone Tissue-Like Environments in PEG Hydrogels," Adv. Healthc. Mater., p. n/a-n/a, 2015.

[16] L. S. M. Teixeira, J. Feijen, C. a van Blitterswijk, P. J. Dijkstra, and M. Karperien, "Enzyme-catalyzed crosslinkable hydrogels: emerging strategies for tissue engineering.," Biomaterials, vol. 33, no. 5, pp. 1281-90, Mar. 2012.

[17] T. Buerli, C. Pellegrino, K. Baer, B. Lardi-Studler, I. Chudotvorova, J.-M. Fritschy, I. Medina, and C. Fuhrer, "Efficient transfection of DNA or shRNA vectors into neurons using magnetofection.," Nat. Protoc., vol. 2, no. 12, pp. 3090-101, Jan. 2007.

[18] G. Palazzolo, N. Broguiere, O. Cenciarelli, H. Dermutz, and M. Zenobi-Wong, "Ultrasoft Alginate Hydrogels Support Long-Term Three-Dimensional Functional Neuronal Networks," Tissue Eng. Part A, vol. 21, no. 15-16, pp. 2177-2185, 2015.

[19] Y. Yu and Y. Chau, "One-step 'click' method for generating vinyl sulfone groups on hydroxylcontaining water-soluble polymers.," Biomacromolecules, vol. 13, no. 3, pp. 937-42, Mar. 2012. 


\section{Supplementary information}

Novel enzymatically cross-linked hyaluronan hydrogels support the formation of 3D neuronal networks

Nicolas Broguiere, Luca Isenmann, Marcy Zenobi-Wong
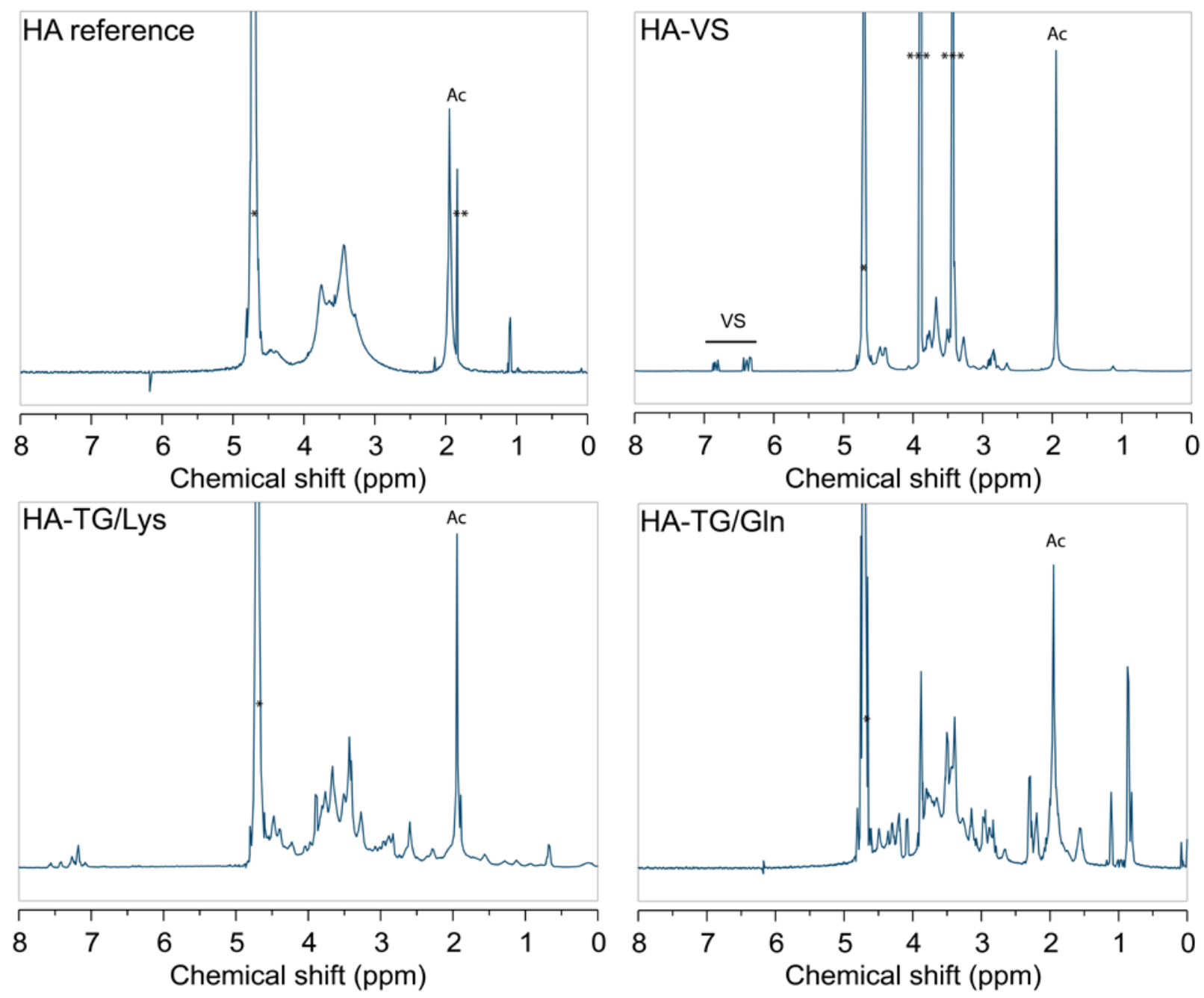

Supplementary Figure 1. NMR spectra of the HA starting material and the VS and TG substrate derivatives. Note the presence of vinyl protons between 6 and 7 ppm on HA-VS, and their complete disappearance after peptide conjugation, showing complete substitution. The $\mathrm{N}$-acetylate from HA backbone (Ac) is used as an internal reference to quantify degrees of substitution with VS. $\left({ }^{*}\right)$ water $\left({ }^{* *}\right)$ acetone $\left({ }^{* *}\right)$ TEOA buffer. 

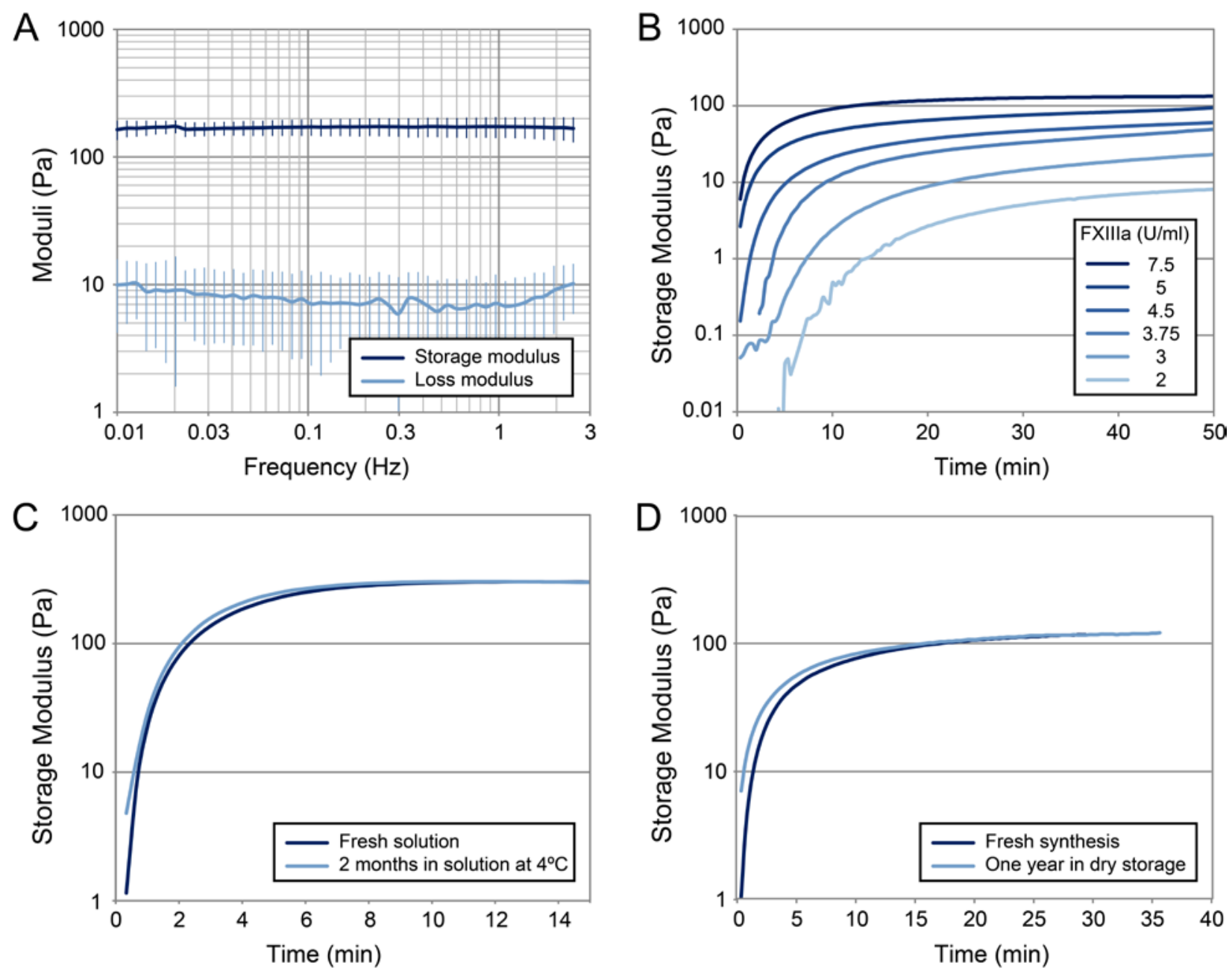

Supplementary Figure 2. Additional mechanical measurements. (A) Frequency response of a 0.5\% HA-TG gel after free swelling for 3 days in PBS. Error bars: $S D n=3$. (B) Influence of the concentration of enzyme on the gelling speed of $0.5 \%$ HA-TG gels. (C) Comparison of the gelling curve of $1 \%$ HA-TG for a fresh solution versus a solution that has been kept for 2 months showing stability in solution. (D) Comparison of the gelling curve of $0.5 \%$ HA-TG for a freshly synthesized HA derivative versus one kept frozen in dry form for 1 year showing stability in storage. 